\title{
Operation stability of radiosonde super- regenerative transceivers for upper-air sounding
}

\author{
Sergey Kudinov ${ }^{1, *}$, Viacheslav Ivanov ${ }^{1}$, and Andrei Gusev ${ }^{1}$ \\ ${ }^{1}$ Ural Federal University, 620002, Ekaterinburg, Russia
}

\begin{abstract}
The use of super-regenerative transceivers in a radiosonde of radar sounding systems can significantly reduce the cost of measuring atmospheric meteorological parameters. Methods and technological norms are proposed for implementing the required technical parameters of radiosondes to ensure the stability of the transceivers in serial production and operation.
\end{abstract}

\section{Introduction}

The use of super-regenerative transceivers as part of aerological radiosondes of atmospheric sounding radar systems makes it possible to transmit telemetric information to a groundbased radar and to measure angular coordinates and slant ranges using an active pulse method on a single carrier frequency.

High sensitivity to interrogation radio pulses $(-90 \mathrm{~dB} / \mathrm{W})$ and a large level of average radiated power of a super-regenerative transceiver $(0.2 \mathrm{~W})$ provide an operational radius of the atmosphere sounding system of at least $250 \mathrm{~km}$. The microwave self-oscillator of the super-regenerative transceiver operates in a complex pulse mode, requiring special circuitry and technological solutions to be considered for its support [1-3].

\section{Main features and characteristics of a super- regenerative transceiver}

The structural diagram containing the main nodes of the super-regenerative transceiver is shown in Figure 1. The graphs represent the features of the super-regenerative transceiver is shown in Figure 2: $U^{\text {Radar }}{ }_{T x}-$ this is the envelope of radio pulses with a duration $\tau_{\mathrm{p} \text { req }}$ and a stationary amplitude of the voltage $U_{p}$ req emitted by the radar; $U_{p}$ - this is the envelope of radio pulses with a pulse duration $\tau \mathrm{pm}$ and voltage amplitude $\mathrm{U}_{\mathrm{st}}$ emitted by a superregenerative transceiver; $U_{\mathrm{b}-\mathrm{e}}$ - this is the video pulses of a suppression frequency generator with a repetition period $\mathrm{T}_{\text {sup }}$ and pulse duration $\tau_{\text {sup }} ; U_{\mathrm{b}}$ - this is the voltage at the baseemitter junction of the transistor of the microwave oscillator of the super-regenerative transceiver, corresponding to the boundary current $\mathrm{I}_{\mathrm{b}} ; \Delta U_{\mathrm{b}-\mathrm{e}}-$ this is the voltage increment

*Corresponding author: kudinoffs@mail.ru 
at the output resistance of the suppression frequency generator due to the base current of the transistor of the microwave oscillator of the super-regenerative transceiver; $\delta(t)$ - this is the law of variation of the attenuation decrement of the oscillatory circuit of a microwave oscillator of a super-regenerative transceiver; $I_{\mathrm{e} 0}-$ this is the emitter current of the microwave transistor during the action of the pulse of the suppression frequency generator; $\mathrm{I}_{\mathrm{s}}$ - start current of a microwave generator of a super-regenerative transceiver.

The microwave oscillator of the super-regenerative transceiver starts to generate a radio frequency signal at the time of the periodic appearance of a pulse of a suppression frequency generator $U_{\text {b-e }}$ and turns off when it ends in the damping interval $\tau_{\text {demp. The }}$ operating frequency of the microwave oscillator of a superregenerative transceiver of a radiosonde is about $1680 \mathrm{MHz}$. The pulse frequency of the suppression frequency generator is $800 \mathrm{kHz}$ (period $\mathrm{T}_{\text {sup }}=1.25 \mu \mathrm{s}$ ). The oscillatory system of a superregenerative transceiver in the off state is characterized by its own attenuation $\delta_{0}$. The change in the circuit attenuation during the

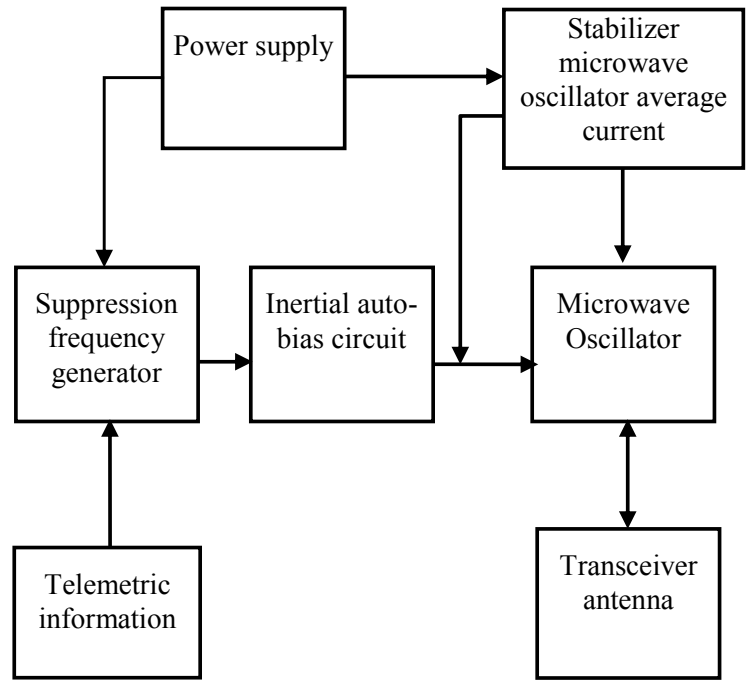

Fig. 1. Block diagram of super-regenerative radiosonde transceiver positive pulse time $\tau_{\text {sup }}$ of the suppression frequency generator determines the process of development and establishment of radio frequency oscillations from the voltage level of fluctuation noise $U_{\mathrm{f}}$ to the voltage level of the stationary mode $\mathrm{U}_{\mathrm{st}}$ The boundary current $\mathrm{I}_{\mathrm{b}}$ corresponds to the attenuation $\delta(t)=0$, the starting current $I_{\mathrm{s}}$ corresponds to the attenuation $\delta(\mathrm{t})=\delta_{\mathrm{s}}[4]$.

To ensure high sensitivity of the super regenerative transceiver in the receiving mode, it is necessary to turn on the microwave oscillator with a minimum value of the starting negative attenuation $\delta_{\mathrm{s}}$. To achieve the maximum output power proportional to $\mathrm{U}_{\mathrm{st}}$ and to quickly establish the amplitude of the radio frequency self-oscillations $U_{p}$ in the microwave generator of the super-regenerative transceiver, it is necessary to realize negative attenuation in the region of medium and large amplitudes of the radio-frequency selfoscillations for this type of microwave transistor. For this, an analysis of the equation of the oscillator of the form was carried out:

$$
\frac{d U}{d t}=-\delta(t, U) U
$$

The study of various options for the implementation of the function $\delta(t, U)$ showed that in order to ensure these conflicting requirements, it is necessary to implement a transient process in the oscillatory circuit of a super-regenerative transceiver with a rigid nature of the establishment of oscillations [3, 4]. In this case, a start of the super-regenerative transceiver can occur with a minimum negative attenuation value at $\delta(\mathrm{t})=\delta_{\mathrm{s}}$, which provides a minimum bandwidth of the oscillatory circuit and high sensitivity in the receiving mode. A stationary mode of self-oscillations is established in the region of maximum amplitudes $U_{\text {st }}$ and a high level of output radiated power in the transmitting mode. In a super-regenerative transceiver, the start of the microwave oscillator should in principle be carried out in the mode of suppressing shock oscillations arising in the 
oscillatory circuit. The leading edge of the radio pulses fluctuates due to the influence of noise at the time the super-regenerative transceiver is started, which indicates a high sensitivity to an external signal in receive mode [5]. As a result of our research, the following was established - to ensure a transient process with a rigid nature of the establishment of oscillations in the oscillatory circuit of a microwave oscillator, it is necessary to apply voltage pulses from a generator of a frequency generator with a low output resistance to the base circuit of a microwave transistor, while the gain of the microwave transistor in power should be the maximum possible.

The super-regenerative amplification effect is reduced by reducing the delay time $\tau \mathrm{dm}$ from the leading edge of the microwave pulses of the microwave oscillator relative to the leading edge of the superimposing frequency pulses by $\Delta \tau \mathrm{d}$ when an external signal $U_{p}$ req appears during the receiving operation interval $\tau_{\mathrm{Rx}}$ adjacent to the start of the superregenerative transceiver. Accordingly, the duration of the emitted radio pulse and its energy increase. The occurrence of selfoscillations in the oscillatory circuit occurs when damping $\delta_{\mathrm{s}}>0$, which is determined by the inrush current exceeding the value of the boundary current $I_{s}>I_{b}$ at the time of the start of the oscillator. Therefore, a change in the value of the start current $I_{s}$ leads to a change in the delay time $\tau \mathrm{dm}$ and the increment of the delay time $\Delta \tau_{\mathrm{d}}$. The dynamic range of the amplitude of the
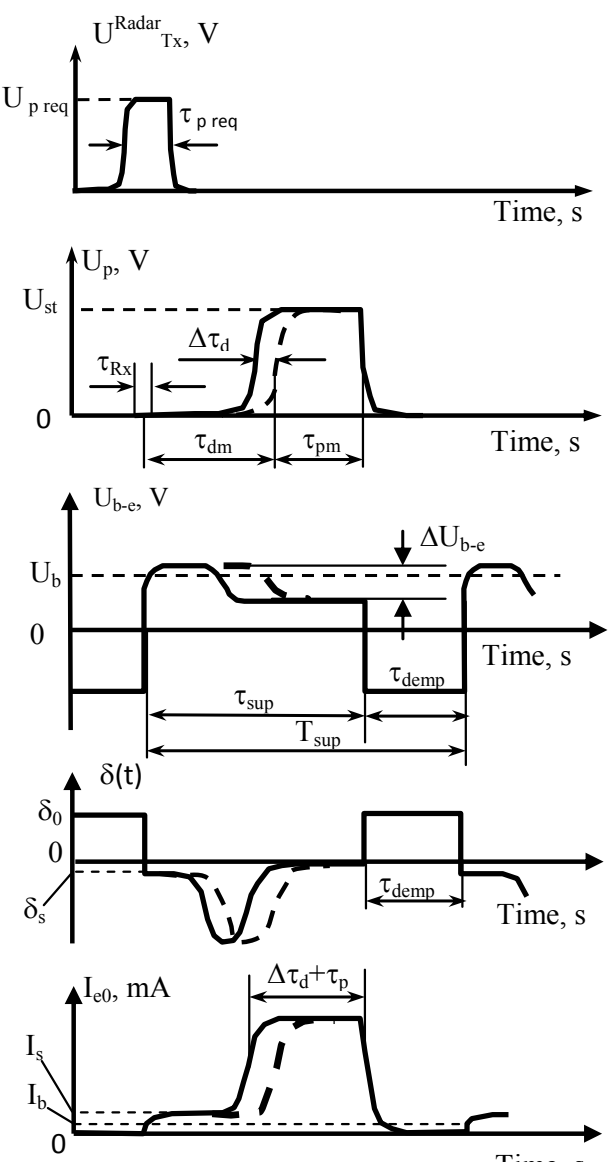

Time, s

Fig. 2. Changing the duration of the emitted radio pulse of a super-regenerative transceiver when receiving a request signal from a radar. radio frequency oscillations in the super-regenerative transceiver circuit is 100-120 dB. Thus, the super-regenerative transceiver is a device with a temporary separation of the receiving and transmitting operating modes during one period of the supercharging frequency $\mathrm{T}_{\text {sup. }}$. As a result of our research, a node for stabilizing the average current of a microwave oscillator was developed, which provides automatic adjustment of the starting current $I_{s}$, supports the required value of the average duration of the emitted radio pulses $\tau_{\mathrm{pm}}$, provides high sensitivity values in the receiving mode and radiated power in the transmitting mode.

A feature of the super-regenerative transceiver is the use of an inertial auto-bias circuit of a microwave oscillator. A capacitor circuit of the auto-bias circuit $\mathrm{C}_{\mathrm{ab}}$ is connected between the output of the generator of the superficial frequency and the input of the microwave oscillator. The inertial auto-bias circuit is necessary to ensure the operating mode with a "response pause" when measuring the range to the radiosonde as part of the atmospheric radiosonde system. The value of the time constant is determined by the ratio

$$
T_{a b}=\left(R_{o u t}+R_{e q}\right) C_{a b},
$$


where Rout is the output impedance of the generator of the superficial frequency; Req equivalent input impedance of a microwave transistor; $\mathrm{Cab}$ is the capacitance of the capacitor of the auto-bias circuit of the microwave oscillator. When a requesting radio pulse arrives at the time the microwave oscillator is started (see Fig. 2), the delay time of the radio pulse $\tau \mathrm{dm}$ is reduced by a value of $\Delta \tau \mathrm{d}$, and its duration and energy increase. This leads to an additional charge of the capacitor of the auto-bias circuit and a corresponding decrease in the inrush current Is at the start of the next radio pulse until its suppression. The tracking radar determines the range at the pause mark in the received radiosonde signal. However, the use of an inertial auto-bias circuit to generate a response signal in the form of a "pause" complicates the setup and operation of a super-regenerative transceiver in operating conditions and requires special research $[4,5]$.

\section{Stability of super-regenerative transceiver operating modes}

From the above materials it follows that in order to determine the conditions for the stable operation of a super-regenerative transceiver, it is necessary to establish a quantitative relationship between the mutual influence of fluctuation noises, the level of the radar request signal at the input, the starting current, the time constant of the inertial auto-bias circuit, and other operating parameters of the microwave oscillator.

It is necessary to introduce into consideration the ratios for calculating the main parameters of the receiving mode of the super-regenerative transceiver. We can write down the formula for calculating $\tau \mathrm{dm}$ using the previously obtained research results [6-8], in the following form:

$$
\tau_{d m}=\frac{1}{\left|\delta_{\mathrm{s}}\right|}\left[\ln \frac{0.1 \mathrm{U}_{\mathrm{st}}}{U_{f}}-\ln \frac{U_{s c}}{U_{f}}+\frac{1}{2} E_{i}\left(-\frac{U_{s c}^{2}}{U_{f}^{2}}\right)\right],
$$

where $U_{\mathrm{sc}}$ is the amplitude of the external signal in the super-regenerative transceiver loop; $U_{\mathrm{f}}$ is the amplitude of fluctuations in the circuit; $E_{\mathrm{i}}$ is an integral exponential function.

Formula (3) can be reduced to a more convenient form for analysis at small levels of the external signal $U_{\mathrm{sc}} \rightarrow 0$ and upon transition to the decimal logarithm:

$$
\tau_{d m} \cong \frac{2,3(m-1)}{\left|\delta_{\mathrm{s}}\right|}
$$

where $m=\lg \frac{0,1 U_{\text {st }}}{U_{\mathrm{f}}}$.

In the general case, the average delay time $\tau_{\mathrm{dm}}$ can change either under the action of an external signal that changes the parameter " $\mathrm{m}$ ", or due to a change in the starting attenuation $\left|\delta_{\mathrm{s}}\right|$. The differential delay time increment $\tau_{\mathrm{dm}}$ can be represented as follows:

$$
d \tau_{d m}=2,3\left(\frac{d m}{\left|\delta_{\mathrm{s}}\right|}-\frac{m-1}{\left|\delta_{\mathrm{s}}\right|^{2}} d\left|\delta_{1}\right|\right)
$$

The first term in formula (5) describes the primary response of an over regenerative transceiver. The second term describes the delay time variation with the attenuation variation. The second term in formula (5) describes the secondary reaction of the superregenerative transceiver if the cause of the change in the starting attenuation $\left|\delta_{\mathrm{s}}\right|$ is the primary reaction. The formula for calculating the primary reaction can be written in the form, passing to the final increments: 


$$
\Delta \tau_{\mathrm{d}}^{\prime}=\tau_{\mathrm{dm}} \frac{m-m^{\prime}}{m-1}
$$

where $m^{\prime}=\lg \frac{0,1 \mathrm{U}_{\mathrm{st}}}{U_{\mathrm{f}}+U_{s c}}$.

The secondary reaction is associated with a change in the starting current when starting the generation of the next radio pulse. The instantaneous value of the starting current of the next radio pulse will depend on the duration of the previous radio pulse, since the time constant of the auto-bias circuit is $\mathrm{T}_{\mathrm{ab}} \geq \mathrm{T}_{\text {sup. }}$. The level of the secondary reaction in the form of a change in time can be determined using the transmission coefficient of the auto-bias circuit $K_{\mathrm{b}}$ of the microwave oscillator [4]:

$$
\Delta \tau_{\mathrm{d}}^{\prime \prime}=\tau_{\mathrm{dm}} K_{b} \Delta \tau_{d}
$$

where $K_{\mathrm{b}}=\frac{1}{\varphi_{T}} \frac{1}{C_{\mathrm{a} b}}\left(I_{\mathrm{bp}}+I_{\mathrm{bs}}\right)$, where $I_{\mathrm{bp}}$ is the pulse current of the base, $I_{\mathrm{bs}}$ is the starting current of the microwave oscillator; $\varphi_{\mathrm{T}}$ is the temperature potential.

The expression connecting the delay time of the previous radio pulse $\tau_{\mathrm{di}}$ with the subsequent $\tau_{\mathrm{d}(\mathrm{i}+1)}$ for further analysis is advisable to write down taking into account the regenerative parameters of the microwave oscillator $[9,10]$ :

$$
\tau_{\mathrm{d}}^{\prime \prime}=\frac{4,6 C_{c}(m-1)}{\omega C_{\mathrm{c}-\mathrm{e}}\left\{\operatorname{Im} \dot{\alpha}_{\mathrm{s}}-\chi_{\mathrm{n}} \cdot \mathrm{I}_{\mathrm{sm}} \cdot\left[K_{\mathrm{b}} \cdot\left(\tau_{\mathrm{dm}}-\tau_{\mathrm{d}}^{1}\right)\right]\right\}},
$$

where Im $\alpha$ is the imaginary component of the complex emitter current transfer coefficient in a circuit with a common base; $\chi_{\mathrm{s}}=\frac{d \operatorname{Im} \dot{\alpha}}{d I_{\mathrm{e} 0}}$ when $\mathrm{I}_{\mathrm{e} 0}=\mathrm{I}_{\mathrm{s} \mathrm{m}}$; where $\mathrm{I}_{\mathrm{s} \mathrm{m}}$ - the average value of the starting current in a stable mode of operation; $C_{\text {c-e }}-$ collector - emitter capacitance; $C_{\mathrm{c}}$ - total capacitance of the super-regenerative transceiver circuit.

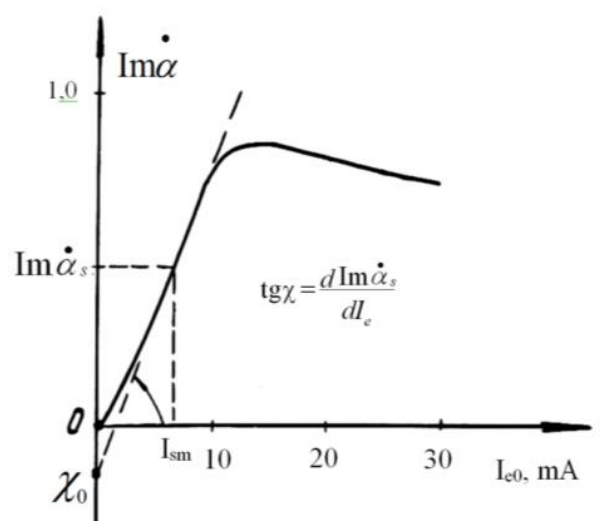

Fig. 3. Linear approximation of the imaginary component of the complex emitter current transfer coefficient in the starting mode of operation of a super-regenerative transceiver.

Since the starting current $I_{s}$ in the operating mode varies very slightly, within a few percent of the nominal value, therefore, the dependence of Im $\alpha$ on the emitter current $\mathrm{I}_{\mathrm{e} 0}$ in the vicinity of $\mathrm{I}_{\mathrm{s}}$ can be approximated by a linear function (Fig.3.)

$$
\operatorname{Im} \dot{\alpha}=\operatorname{Im} \dot{\alpha}_{\mathrm{s}}+\chi_{\mathrm{s}} \cdot\left(\mathrm{I}_{\mathrm{e} 0}-\mathrm{I}_{\mathrm{sm}}\right) .
$$

It should be noted that the inrush current $\mathrm{I}_{\mathrm{S}(\mathrm{i}+1)}$ will also additionally randomly change due to fluctuations $\tau_{\mathrm{di}}$ and the effect of charge fluctuations of the auto-bias circuit when the conditions $\quad K_{\mathrm{b}}>0$, are fulfilled.

A characteristic violation of the stable operation of a super-regenerative transceiver is manifested in the fact that one sequence of radio pulses has a duration longer than the duration of another sequence. The parameters of the extended radio pulses are indicated by the index $i$, and the parameters of the radio pulses with a reduced duration value by the index $(i+1)$ is shown in Figure 4. 
This mode of operation leads to a decrease in the sensitivity and level of the response signal of the super-regenerative transceiver, a significant decrease in the efficiency of transmitting telemetric information to the radar [11]. Next, a statistical approach will be used to evaluate its main characteristics to take into account the significant influence of fluctuation noise on the operation mode of a superregenerative transceiver.

As noted earlier, the value $\tau \mathrm{md}$ is probabilistic due to fluctuations in the initial conditions. In the event that there is no mutual influence between the radio pulses $\left(K_{\mathrm{b}}=0\right)$, the law of the distribution of the delay time $W\left(\tau_{\mathrm{d}}\right)$ can be reduced to the form $[6,7]$

$$
W\left(\tau_{\mathrm{d}}\right)=\frac{2 K_{1}^{2}}{\left|\delta_{\mathrm{s}}\right|} \exp \left\{-\left[2\left|\delta_{\mathrm{s}}\right| \tau_{\mathrm{d}}+K_{1}^{2} \exp \left(-2\left|\delta_{\mathrm{s}}\right| \tau_{\mathrm{d}}\right)\right]\right\}
$$

where $K_{1}=\frac{0.1 U_{\text {st }}}{\sqrt{2} U_{\mathrm{f}}}$.

The distribution density graph $W\left(\tau_{\mathrm{d}}\right)$ for this case is shown in Figure 5. In this case, the variance of the delay time is determined by the starting value of the circuit attenuation and, accordingly, can be determined by the formula:

$$
\sigma_{\tau}^{2}=\frac{\pi^{2}}{\left|\delta_{s}\right|^{2} 24}
$$

In the "secondary reaction" mode, the transmission coefficient of the auto-bias circuit should be $K_{\mathrm{b}}>0$, since it is necessary to provide the necessary level of the secondary reaction, i.e. strive to increase the parameter $K_{\mathrm{b}}$. In this case, the value of the starting current for (i+1)-pulse can be expressed in terms of the instantaneous value of the delay time for i-pulse using the expression:

$$
I_{\mathrm{s}(i+1)}=\mathrm{I}_{\mathrm{sm}}\left[1-K_{\mathrm{b}}\left(\tau_{\mathrm{dm}}-\tau_{\mathrm{d} i}\right)\right] .
$$

The analysis shows that the variance $\sigma_{\tau}{ }^{2}$ of the distribution law for $\tau_{\mathrm{d}}$ will increase monotonically with increasing parameter $K_{\mathrm{b}}$.

It can be assumed, that the instantaneous values of the delay time $\tau_{\mathrm{di}}$ will be grouped around their mathematical expectation $W\left(\tau_{\mathrm{dmi}}\right)$, and the values of the delay time $\tau_{\mathrm{dmi}}$, respectively, will have the mathematical expectation $\mathrm{W}\left(\tau_{\mathrm{dm}(i+1)}\right)$ due to the effects of the inertial circuit of auto-bias at $\mathrm{T}_{\mathrm{ab}} \geq \mathrm{T}_{\text {sup }}$. Therefore, with an increase in the $K_{\mathrm{b}}$ coefficient, the initial distribution law of random variables for $i$ - and $(i+1)$-sequences of radio pulses (see Figure 5) will be transformed into different distribution laws for $i-$ and $(i+1)$-sequences of radio pulses (Figure 6). In this case, a balance of the charge flowing through the capacitor of the auto-bias circuit $C_{\mathrm{ab}}$ is performed for a time interval equal to twice the value of the suppression period, etc. It is necessary to analyze the operation of a super-regenerative 
transceiver based on a system of stochastic differential equations for an accurate stability study. However, without complicating the study of the issue, the basic conditions for the stable operation of a super-regenerative transceiver can be determined in the framework of the statistical approximation.

Regarding the parameters included in formula (8), the following remarks should be made: the quantity " $m$ " is random due to fluctuations in the initial conditions; the starting current Is should also be considered as a random variable, including due to shot-like fluctuations. In this case, the distribution density for delay $\tau_{\mathrm{d}}$ is determined by the distribution law of the independent quantities "m", "I $\mathrm{I}_{\mathrm{s}}$, " $\tau_{\mathrm{dm}}-\tau_{\mathrm{di}}$ ", and the magnitude of the transmission coefficient of the autoblock circuit $K_{\mathrm{b}}$ determines the degree of correlation of the pulses of the super-regenerative transceiver and significantly affects the nature of the distribution law for $\tau_{\mathrm{di}}$ and $\tau_{\mathrm{d}(\mathrm{i}+1)}$. To solve this problem, we used the Monte Carlo statistical modeling method, which allows us to implement transformations of the form $(8$, $10,12)$ and obtain final distributions for $\tau_{\mathrm{di}}$ and $\tau_{\mathrm{d}(\mathrm{i}+1)}$ based on the generation of random numbers modeling random variables " $\mathrm{m}$ " and "I $\mathrm{S}_{\mathrm{s}}$ ". Variables and constants are introduced as initial data for modeling: $\mathrm{m}, \tau_{\text {sup }}, \tau_{\mathrm{dm}}, \mathrm{I}_{\mathrm{sm}}, K_{\mathrm{b}}$, $\mathrm{U}_{\text {st. }}$. To calculate the instantaneous value " $\mathrm{m}$ "

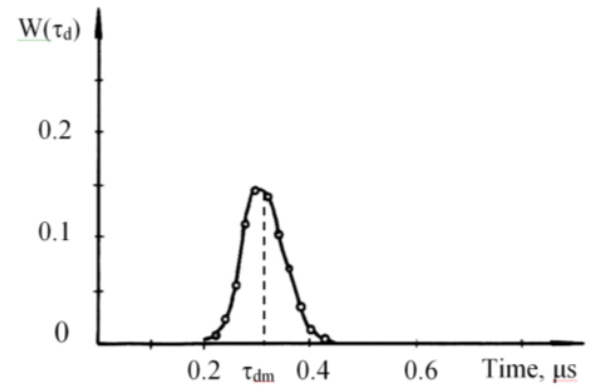

Fig. 5. The law of the distribution of the delay time of $i$ - and $(i+1)$-sequences of emitted radio pulses with a stable mode of operation of a super regenerative transceiver $\left(K_{\mathrm{b}}<2.4 \cdot 10^{5}\right)$. (4), the fluctuations $U_{\mathrm{f}}$ at the start time are represented as the sum of two quadrature components distributed according to the normal law with a mathematical expectation equal to zero $(\mathrm{M} 1=\mathrm{M} 2=0)$ and equal variances $\sigma_{\mathrm{H} 1}^{2}=\sigma_{\mathrm{H} 2}^{2}=\frac{U_{\mathrm{f}}^{2}}{2}$.

The generation of random variables according to the normal law for a given mathematical expectation and variance occurs when accessing the library routine. For the initial value of $\tau \mathrm{di}$ and the instantaneous value of $\mathrm{m}_{(\mathrm{i}+1)}$ according to formulas (10-12), the instantaneous value for $\tau_{\mathrm{d}(\mathrm{i}+1)}$ is calculated, which is then used for the next cycle of calculating the value of $\tau_{\mathrm{d}(\mathrm{i}+2)}$ and etc. At each calculation cycle, a call is made to the standard routine that calculates the new mi values. The number of cycles $\mathrm{N}$ corresponds to the number of radio pulses of the superregenerative transceiver in the observation interval of the distribution law. In practice, the distribution law is established for the number of

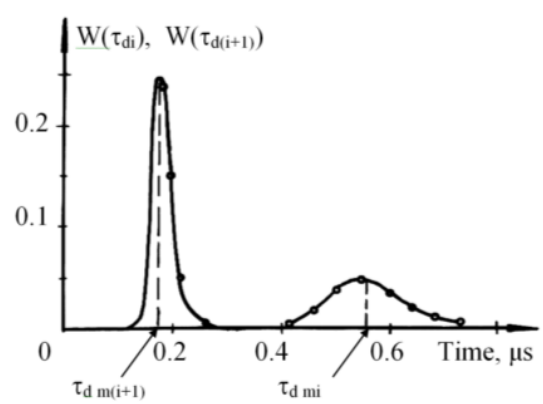

Fig. 5. Distribution laws for i- and (i+1)sequences of radio pulses of a superregenerative transceiver in an unstable mode of operation $\left(K_{\mathrm{b}}=3.6 \cdot 10^{5}\right)$. cycles $\mathrm{N} \geq 500$. The interval of possible values of the delay time $\tau \mathrm{d}$ is equal to the duration of the superimposing pulses. It is divided into $n$ numbered sampling intervals. Even (i+1)and odd (i)-sequences of $\tau$ d values are distributed in two rows R1 (I) and R2 (I), and the relative frequency of occurrence of the $\tau \mathrm{d}$ value corresponding to the number of the sampling interval is determined. The obtained histograms of the distribution of $\tau \mathrm{d}$ for even and odd sequences of radio pulses are taken as the distribution laws $W\left(\tau_{\mathrm{di}}\right), W\left(\tau_{\mathrm{d}(\mathrm{i}+1)}\right)$, (see Figures 5,6). 
The distribution law $W\left(\tau_{\mathrm{d}}\right)$, calculated for a serial super-regenerative transceiver with the parameter $K_{\mathrm{b}}=2.4 \cdot 10^{5}, \mathrm{~s}^{-1}$, is shown in Fig. 5. Since the distribution laws for $\mathrm{i}-$ and $(\mathrm{i}+1)$-sequences of radio pulses practically coincide $\left(\tau_{\mathrm{dmi}}-\tau_{\mathrm{dm}(\mathrm{i}+1)} \approx 0\right)$, the operation mode of the super-regenerative transceiver is considered stable. The calculation results $W\left(\tau_{\mathrm{d}}\right)$ for $K_{\mathrm{b}}=3.6 \cdot 10^{5}, \mathrm{~s}^{-1}$, which show that the distribution laws for even and odd radio pulses are significantly different $\left(\tau_{\mathrm{dmi}}-\tau_{\mathrm{dm}(i+1)} \approx 0.4 \mu \mathrm{s}\right)$ are given in Figure 6 . In this case, the operation mode of the super-regenerative transceiver is unstable.

To determine the maximum value of the auto-bias coefficient $K_{\mathrm{bmax}}$, the permissible fluctuation value $\Delta \tau_{\mathrm{d}}$ in the calculations should be limited to the value $\Delta \tau_{\mathrm{d}} \leq \sigma_{\tau}(11)$, since otherwise the transmission efficiency of telemetric information in the transmitting mode of the super-regenerative transceiver will decrease. The value of $K_{\mathrm{bmax}}$ can be estimated on the basis of the assumption that the random increment of the duration of the i-pulse should not exceed the increment of the duration of the (i+1)-pulse due to the reaction of the auto-bias circuit:

$$
\Delta \tau_{\mathrm{d}(\mathrm{i}+1)} \leq \Delta \tau_{\mathrm{di}}
$$

In practice, we can assume that $\Delta \tau_{\mathrm{d}} \leq \sigma_{\tau}$.

Taking into account relations (7-11) and condition (13), we can obtain a formula for calculating the maximum value of $K_{\text {bmax }}$ (the minimum value of the capacitance of a selfbiasing capacitor, $\mathrm{Cab} \mathrm{min}$ ) at which the operating mode of the super-regenerative transceiver with a secondary reaction can still be considered stable:

$$
C_{\mathrm{ab} \min } \geq \frac{\tau_{\mathrm{dm}} \cdot \chi_{\mathrm{s}} \cdot I_{\mathrm{sm}} \cdot\left(I_{\mathrm{bp}}+I_{\mathrm{bs}}\right)}{\varphi_{\mathrm{T}} \cdot \operatorname{Im} \dot{\alpha}_{\mathrm{s}}} .
$$

Expression (14) correctly takes into account the influence of the main parameters of the super-regenerative transceiver on the condition of stable operation, despite its approximate nature. In particular, it can be seen that with an increase in $\tau \mathrm{dm}$, in order to maintain the stability of the super-regenerative transceiver, it is necessary to increase the capacitance of the capacitor of the auto-bias circuit accordingly. This is confirmed by experimental studies of a super-regenerative transceiver. Evaluation of the average value of the time difference between $\tau_{\mathrm{dmi}}$ and $\tau_{\mathrm{dm}(\mathrm{i}+1)}$ shows that it will not exceed the value of $\Delta \tau_{\mathrm{d}} \leq 2 \sigma_{\tau}$ for the capacitor capacitance $C_{\mathrm{abmin}}$ calculated from condition (14).

A level of secondary reaction, i.e. the real sensitivity of the super-regenerative transceiver in this case will satisfy the technical requirements.

Given the results obtained, it is also possible to determine the conditions for the stable functioning of a super-regenerative transceiver in the primary reaction mode. In this case, the secondary reaction should be completely absent with the value of the auto-displacement coefficient tending to zero $\left(K_{\mathrm{b}} \rightarrow 0\right)$. In practice, this can be done by increasing the capacitance of the capacitor $C_{\mathrm{ab}}$ of the auto-bias circuit. A setting parameters of a superregenerative transceiver in the primary reaction only mode is much simpler than tuning with a secondary reaction, its stability is significantly higher, which is important in operating conditions. In (12), a possibility of using the phase synchronization effect of radio pulses of a super regenerative transceiver in the primary reaction mode when using a coherent radar interrogation signal to measure the range and velocity of a radiosonde was discussed.

\section{Conclusion}

The effect of the fluctuations of the leading edge of the radio pulses and the transfer coefficient of the inertial auto-bias circuit of the microwave oscillator on the sensitivity and stability of the super-regenerative transceiver is studied. The factors determining the 
stability of the super-regenerative transceiver in the mode of primary and secondary responses to the radar query signal are determined.

To increase the stability of the super-regenerative transceiver and the operational characteristics of the atmospheric radio sounding system, it is advisable to improve the production technology and methods of digital processing of the response signal in the form of the primary reaction of the super-regenerative transceiver.

\section{References}

1. V.N.Kiselev, A.D. Kuznetsov, Methods of sounding the environment (atmosphere). (Russian State Hydrometeorological University, 2004).

2. M.K. Belkin, G.I. Kravchenko, Yu.G. Skorobutov, B.A. Stryukov, Superregenerators. Radio and communications (1983)

3. V.E. Ivanov, A.V. Gusev, K.A. Ignatkov, S.I. Kudinov, I.V. Malyigin, V.Ya. Noskov, O.V. Plohih, V.V. Ryisev, O.A. Chernyih, Uspekhi sovremennoi radioelektroniki, 9, pp. 3-49 (2015)

4. V.E. Ivanov, M.B. Fridzon, S.P. Essyak, Radio sounding of the atmosphere. Technical and metrological aspects of the development and use of radiosonde measuring instruments. (Ekaterinburg. UrO RAN, 2004)

5. S.I. Kudinov, V.E. Ivanov, Investigation of the influence of fluctuation and shock oscillations on the sensitivity of super-regenerative transceiver devices. Ural Radio Engineering Journal. v. 3, 2, pp. 170-195 (2019)

6. V.I. Grigulevich, On the question of the fluctuation nature of the establishment of oscillations in an electronic oscillator. (Radio engineering, v. 1. pp. 65-70, 1959)

7. V.I. Grigulevich, On the fluctuation nature of the spectrum of an electronic oscillator. (Radio engineering. 4. pp. $446-453,1959$ )

8. V.I. Tikhonov, On the fluctuation nature of the establishment of the amplitude of oscillations in the oscillator. (Radio engineering and electronics. v.9, pp. 1262-1267, 1956)

9. V.E. Ivanov, Study of the regenerative characteristics of a transistor microwaveoscillator, Radio-technical systems for locating spatially distributed objects. UPI, 4. pp. 93-99 (1981)

10. V.E. Ivanov, S.I. Kudinov, Generalized regenerative characteristic of a transistor microwave oscillator. Radio altimetry-2010, Yekaterinburg (2010)

11. V.E. Ivanov, Study of the stability of the operation of a super-regenerative transceiver, Radar, navigation, communication, Voronezh State University, v.3. pp. 2182-2194 (2002)

12. V.E. Ivanov, S.I. Kudinov, Study of the range channel of a coherent radar with an onboard super-regenerative transponder, (Microwave equipment and telecommunication technologies, v. 2, Sevastopol, pp. 905-906 (2012) 